\title{
The effect of photodynamic therapy and polymer solution containing nano-particles of Ag /ZnO on push-out bond strength of the sealers AH-Plus and MTA Fillapex
}

\author{
Hamidreza Yavari ${ }^{1}$, Negin Ghasemi ${ }^{2}$, Baharak Divband ${ }^{3}$, Yashar Rezaei ${ }^{4}$, Golchin Jabbari ${ }^{5}$, Salar Payahoo ${ }^{6}$ \\ ${ }^{1}$ DDS, MSc, Associate Professor, Department of Endodontics, Dental Faculty, Tabriz University (Medical Sciences), Tabriz, Iran \\ ${ }^{2}$ DDS, MSc, Assistant Professor, Department of Endodontics, Dental and Periodontal Research Center, Dental Faculty, Tabriz \\ University (Medical Sciences), Tabriz, Iran \\ ${ }^{3}$ Assistant Professor, Department of Chemistry, Tabriz University, Tabriz, Iran \\ ${ }^{4}$ Assistant Professor, Department of Dental Biomaterials, Faculty of Dentistry, Tabriz University of Medical Sciences, Tabriz, \\ Iran \\ ${ }^{5}$ DDS, Endodontic Department, Dental and Periodontal Research Center, Faculty of Dentistry, Tabriz University of Medical Scien- \\ ces, Tabriz, Iran \\ ${ }^{6}$ DDS, Department of Oral and maxillofacial radiology, Dental and Periodontal Research Center, Faculty of Dentistry, Tabriz Uni- \\ versity of Medical Sciences, Tabriz, Iran
}

Correspondence:

Faculty of Dentistry

Tabriz University of Medical Sciences

Golgasht Ave, Tabriz, IRAN

Jabbarign@gmail.com

Received: 22/05/2017 Accepted: 19/07/2017

\begin{abstract}
Yavari H, Ghasemi N, Divband B, Rezaei Y, Jabbari G, Payahoo S. The effect of photodynamic therapy and polymer solution containing nanoparticles of $\mathrm{Ag} / \mathrm{ZnO}$ on push-out bond strength of the sealers AH-Plus and MTA Fillapex. J Clin Exp Dent. 2017;9(9):e1109-14.

http://www.medicinaoral.com/odo/volumenes/v9i9/jcedv9i9p1109.pdf
\end{abstract}

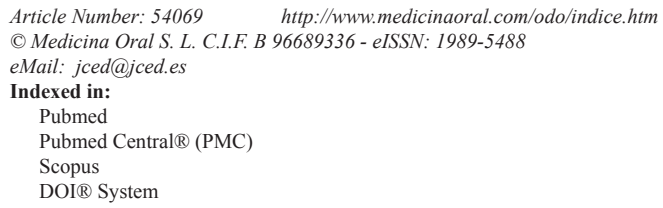

\begin{abstract}
Background: The aim of this in vitro study was to examine and compare the effect of photodynamic therapy and solution containing nano particles $\mathrm{Ag} / \mathrm{ZnO}$ on adhesion of endodontic sealers to dentinal walls of human root canal. Material and Methods: Ninty single-rooted human teeth were selected and their clinical crown was cut from the cemento-enamel junction zone. Canals were prepared by RaCe rotary system and the smear layer was removed using $17 \%$ EDTA and 5.25\% NaOCl. Samples were randomly divided into two groups of AH Plus and MTA Fillapex based on the sealer type and each group based on antimicrobial method was divided into two sub-groups of photodynamic therapy and polymer containing nano particles of $\mathrm{Ag} / \mathrm{ZnO}$ and a control sub-group $(\mathrm{N}=15)$. After obturation of canals with gutta-percha and sealers mentioned, the samples were incubated for a week at a humidity of $95 \%$ and $37^{\circ} \mathrm{C}$ and then $2 \mathrm{~mm}$ thick discs were prepared from the middle region of roots for Push-out test. The maximum failure force was recorded in newton and converted to MPa. Then, 3 random specimens of each subgroup were evaluated by scanning electron microscopy. Statistical analysis was performed by Two way ANOVA and ( $P$ $<0.05$ ) was considered significant.

Results: The highest $(4.84 \pm 1.63)$ and lowest $(0.16 \pm 0.075)$ push-out bond strength values were obtained in $(\mathrm{AH}$ Plus-PDT) and (MTA Fillapex-Ag/ZnO) respectively. Independent of antimicrobial method, AH Plus bond strength was significantly higher than the MTA Fillapex $(p<0.001)$. For both sealers, PDT increased the bond strength significantly $(p<0.001)$.
\end{abstract}


Conclusions: Photodynamic therapy has a positive effect on the bond strength of AH Plus and MTA Fillapex sealers.

Key words: AH Plus, MTA Fillapex, Nano particles, Photodynamic therapy, Push-out.

\section{Introduction}

Obturation of root canal system is an essential part of success in endodontics as it aims to close the leakage pathways from the coronal and apical directions(1). The most common material used for root canal obturation is gutta-percha with sealer. An ideal sealer should completely cover the canal space and adhere to the root canal wall and to the gutta-percha (2). Higher bond strength of sealer decreases leakage and also improves the stability of root canal filling materials (3). Based on research, adhesion of sealers to dentin is influenced by several factors including: chemical and physical characteristics of the sealer and its reaction with dentin, irrigation method and the smear layer removal (4-9).

The main purpose of root canal treatment is to remove microorganisms and their byproducts from root canal system and to prevent re-infection (10). One of the new methods introduced for canal disinfection is the use of metal- or polymer-based nanoparticles. Nanoparticles (NPs) are insoluble particles measuring less than 100 $\mathrm{nm}$ (11) that, the high ratio of surface area to volume gives them unique biological, physical and chemical characteristics. Biocompatibility, broad-spectrum and long lasting antibacterial activity are some of their advantages $(12,13)$. Recently, photodynamic therapy (PDT) has been used to eliminate microorganisms of the root canal system in vitro and in vivo (14). This method is able to eliminate cultivable bacteria species especially Enterococcus faecalis from the canal and also to reduce the occurrence of bacterial resistance (15).

AH Plus is a resin-based sealer with widespread use which is biocompatible and has acceptable physical properties. In previous studies, the positive impacts of triple antibiotic paste (16), removing the smear layer $(8,17)$, activating irrigation solution with ultrasonic(18), applying Er:YAG laser (7) on the bond strength of this sealer have been reported. Recently, the sealers with MTA base were introduced to achieve good biological characteristics of MTA. MTA Fillapex is one of these Sealers which is bactericidal and biocompatible (19). The positive impact of the smear layer removal (8) and relative humidity of dentin (6) on bond strength of this sealer have been shown.

Several studies have been conducted on the antimicrobial effectiveness of NPs and PDT but their impact on the dentin surface and adhesion of sealers has been less attended. The aim of this study was to evaluate the impact of these two methods on the adhesion of two types of resin-based and bioceramic sealers using the Push out test.

\section{Material and Methods}

-Synthesis of $\mathrm{Ag} / \mathrm{ZnO}$ nanoparticles

$3.25 \mathrm{~g} \mathrm{Zn}(\mathrm{NO} 3) 2$ was dissolved in $15 \mathrm{ml}$ of ethanol and $40 \mathrm{ml}$ of distilled water. The mixture solution was moved to an ultrasonic bath and the aqueous solution of AgNO3 was dropped into the solution for 90 minutes. The suspension was transferred to a $200 \mathrm{~mL}$ teflon coated autoclave and kept at $120^{\circ} \mathrm{C}$ for $8 \mathrm{~h}$. After the hydrothermal reaction, the precipitate separated from solution by centrifugation at $2000 \mathrm{rpm}$ for $15 \mathrm{~min}$ and washed with deionized water several times and dried at $100^{\circ} \mathrm{C}$ overnight for further characterization. The obtained nanoparticles were dispersed in a polymer solution and the polymerization took place by increasing the temperature. Synthesized $\mathrm{Ag} / \mathrm{ZnO}$ nanoparticles were characterized by Scanning electron microscopy MRIA3-FEG-SEM ( Tescan, Brno, Czech) and Transmission electron microscopy (Zeiss LEO 912 Omega) (Fig. 1).

-Tooth selection and preparation

90 extracted single-canal, closed apex, non-calcified and without curvature human maxillary central incisors teeth (due to periodontal problems) were selected. The removal of the calculus and soft tissue from root surfaces was done by ultrasonic device (Cavitron, Dentsply Ltd,Weybridge, UK). Samples were stored in a $0.5 \%$ chloramine-T solution until the time of the study. The crowns were sectioned from Cemento-enamel junction level using a diamond disc (SP 1600 Microtome, Leica, NuBlock, Germany ). The working length (WL) was determined by using \# 15 K-flexofile (Dentsply Mailefer, Ballaigue, Switzerland) at $1 \mathrm{~mm}$ shorter than the apical foramen.Canal preparation was done with $\mathrm{RaCe}$ rotary system (FKG Dentaire, La-Chaux de Fonds, Switzerland) as follows: \# 40/ 0.10 for the coronal third, \# 35/ 0.08 for the middle third and \# 30/0.06 up to WL. Normal saline was used during instrumentation and finally smear layer was removed using $\mathrm{NaOCl}$ (5.25\%, 3 minutes) and EDTA (17\%, 3 minutes). Then samples were randomly divided into two groups based on the sealer type and each group based on the antimicrobial method used were divided into two experimental sub-groups and one control group. In groups of disinfection with NPs, at the end of preparation $2 \mathrm{~mL}$ of polymer containing $\mathrm{Ag} /$ $\mathrm{ZnO}$ was used as a final rinse for 5 minutes.

In groups of PDT, this method was used as the last step of disinfection. In a dark room the canals were filled with a filter -sterilized solution of toluidine blue $(2 \%$ aq.solution) for 5 minutes. Then light was applied into canals by using a diode laser (Handy laser sprint dental, RJ-laser, Winden, Germany). The wavelength of device 



Fig. 1: Photomicrographs of $\mathrm{Ag} / \mathrm{ZnO}$ nanoparticles: A) SEM, B) TEM.

was $638 \mathrm{~nm}$ and output power was $150 \mathrm{~mW}$. According to the manufacturer instruction light was applied as follows: 2.5 minutes of irradiation, 2.5 minutes of stop and 2.5 minutes of re-radiation to reactive free radicals without increasing the destructive temperature. For a 360degree uniform radiation, a flexible plastic optical fiber (PACT, Cumdente, Tübingen, Germany) with a diameter of 200 micrometers and taper of 0.03 with cylindrical distributors were used. By placing this fiber in $3 \mathrm{~mm}$ of working lenght, guiding light to the entire length of the canal and to the inaccessible areas such as fins and branches were ensured. After irradiation, canals were rinsed with $10 \mathrm{ml}$ of normal saline.

In the control groups no adjunct method was used. Finally, the root canals were dried with paper points and obturated with gutta-percha and AH Plus (Dentsply, Dentrey, Kostanz, Germany) and MTA Fill apex (Angelus, Londrina, PR, Brazil) sealer using lateral condensation technique. Samples were stored at $95 \%$ humidity and $37^{\circ} \mathrm{C}$ for a week and then $2 \mathrm{~mm}$-thick dental disks from the middle third were prepared using a diamond disc with water coolant.

-Push-out bond strength test

Push-out testing was conducted by Universal testing machine (Model H5K-S; Hounsfield Test Equipment, Surrey, England). The force was applied in apico-coronal direction to the samples by using a $1.1 \mathrm{~mm}$ diameter cylindrical plunger parallel to the long axis of the tooth and at crosshead tip speed of $1 \mathrm{~mm} / \mathrm{min}$. The Maximum force which caused the bond failure and displacement of filling material was recorded in Newton $(\mathrm{N})$. Push-out bond strength is obtained in MPa by dividing the load in $\mathrm{N}$ by the area of the bonded interface. The bonded area of each section was calculated using the following formula: (Fig. 2).

$$
\text { Area }=\pi(R+r) \sqrt{ }\left[h^{2}+(R-r)^{2}\right]
$$

Fig. 2: Formula.
Where $\mathrm{R}$ is the coronal radius and $\mathrm{r}$ is the apical radius of disc, $\mathrm{h}$ is disc thickness and $\pi=3.14$.

-Preparations for SEM evaluating

3 specimens of each group were randomly selected to scan with a scanning electron microscope MRIA3-FEGSEM ( Tescan, Brno, Czech), to investigate the bond failure mode. Longitudinal grooves were created at two surfaces of the dental disks symmetrically by a diamond disc without entering the canal space and two pieces were separated by a hammer and chisel. Then the sections produced were coated by gold as a conductor of electric current and scanning electron micrographs were prepared.

-Statistical analysis

Data analysis by kolmogorov-smirnov test showed normal distribution of data. Two- way ANOVA analysis was used to assess the effect of the type of sealer and disinfection method on bond strength to dentin. Post hoc Tukey and independent t-tests were used for pair-wise comparisons of groups. Statistical significance was defined at $p<0.05$.

\section{Results}

The mean and standard deviation of bond strength for the studied groups have been shown in Table 1.The highest and lowest mean push-out bond strength values were obtained in (AH Plus-PDT) and (MTA fillapex- $\mathrm{Ag} / \mathrm{ZnO}$ ) respectively. Regardless of antimicrobial method, AH Plus sealer had higher bond strength than MTA Fillapex in all groups $(p<0.001)$. PDT method significantly increased the bond strength of both sealers $(p<0.001)$. Nano disinfection method had significantly negative effect on the bond strength of AH Plus sealer $(P<0.001)$, but for MTA Fillapex sealer the difference between the NPs and the control group was not significant $(p=0.071)$. In SEM evaluations, the failure patterns for AH Plus sealer were cohesive and for MTA Fillapex were mixed pattern (Fig. 3). Penetration of AH plus 
Table 1: (Mean $\pm \mathrm{SD})$ bonding strength of sealers to dentin.

\begin{tabular}{|l|c|c|}
\hline & AH Plus & MTA Fillapex \\
\hline NPs & $(1.00 \pm 0.39)$ & $(0.16 \pm 0.075)$ \\
\hline PDT & $(4.84 \pm 1.63)$ & $(0.49 \pm 0.16)$ \\
\hline control & $(3.18 \pm 0.48)$ & $(0.26 \pm 0.11)$ \\
\hline
\end{tabular}
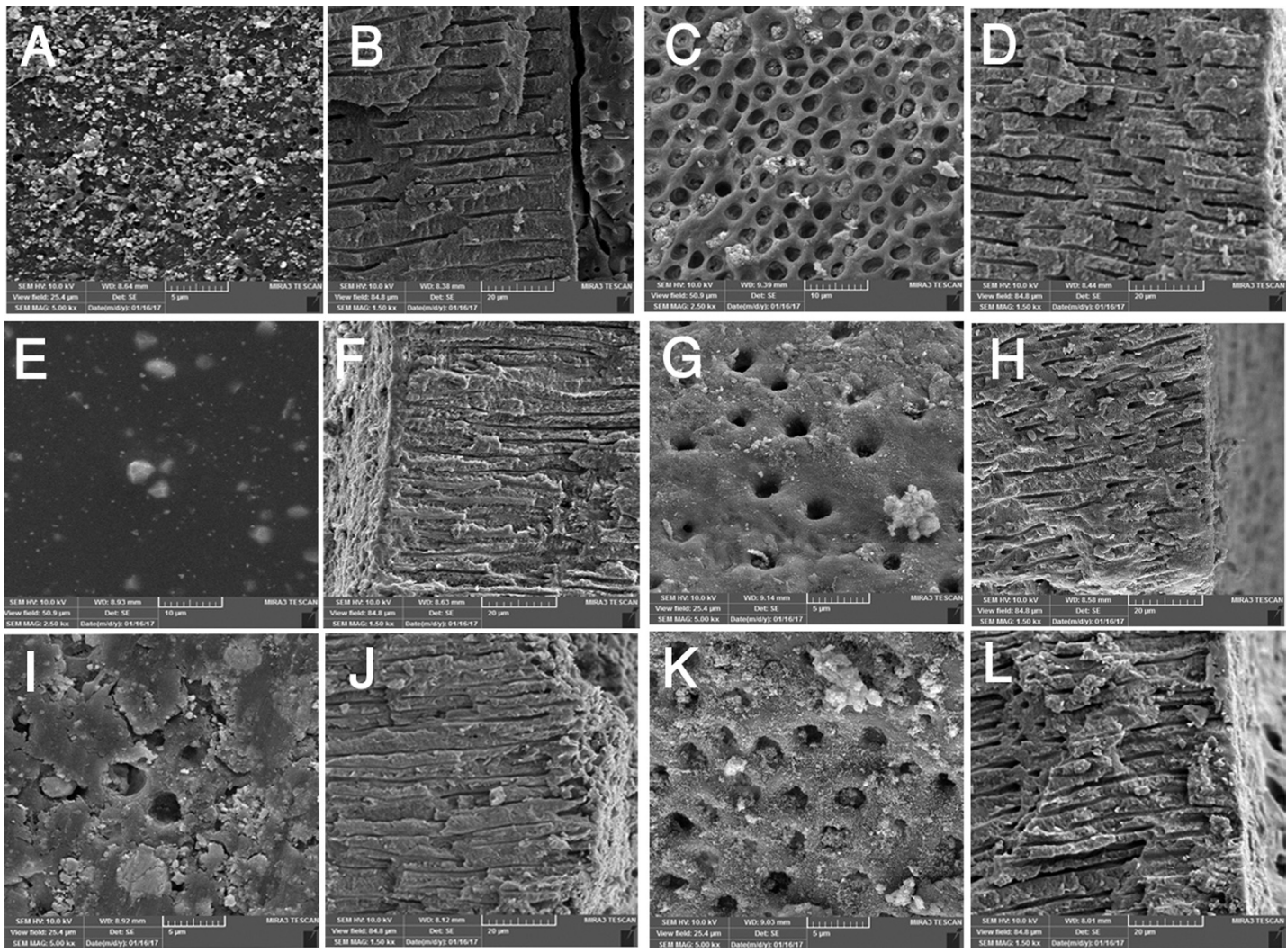

Fig. 3: SEM photomicrogrographs of the studied groups. ( A and B) NPs and AH Plus, (C and D) NPs and MTA Fillapex, (E and F) PDT and AH Plus, (G and H) PDT and MTA Fillapex, (I and J) control and AH Plus, (K and L) control and MTA Fillapex.

sealer into dentinal tubules was observed generally and deep penetration was observed in PDT group (Fig. 3F). Despite the presence of open dentinal tubules MTA fillapex did not penetrate into the most (Fig. 3C,G,K).

\section{Discussion}

This study has compared the bond strength of AH plus and MTA Fillapex sealers to the root canal dentin after using the final disinfection protocol including PDT and antibacterial NPs. PDT method unlike NPs, had a positive effect on the bond strength of both sealers.

Adhesion is one of the basic features for root canal sealers $(3,20)$ and is essential to maintain the integrity of sealer-dentin interface during mechanical stresses applied due to occlusion forces and restorative processes such as post space preparation (21-23). Filling ma-
In this study, two different resin-based and calcium silicate-based sealers have been used and $\mathrm{AH}$ plus sealer showed greater adhesion to radicular dentin in comparison to MTA fillapex. AH Plus has been used as the gold standard of sealers and as the control group in previous studies (27). MTA Fillapex is one of the recently introduced calcium silicate-based sealers which was produced to have the favorable characteristics of the MTA, and for compensation of the features that makes MTA undesirable as a sealer such as working time, setting time and hard handling (19).

SEM images with high magnification allows for highly detailed observation of sealer adaptation and penetration into dentinal tubules (28) and the failure mode due to push out test can be investigated in details. In this study failure modes were cohesive for AH Plus sealer and 
mixed pattern for MTA Fillapex (Fig. 3F). The cohesive failure represents a strong bond between $\mathrm{AH}$ plus and radicular dentin which confirms the push-out test values. Sealer penetration into dentinal tubules and tag formation was observed generally. In MTA fillapex samples, dentinal tubules were visible in adhesive failure areas and although the orifices were open, sealer did not well penetrate all tubules (Fig. 3C,G,K). This pattern have been reported for other sealers previously $(7,29)$.

Higher bond strength for AH Plus compared to MTA Fillapex has been reported in the litreture $(6,30)$ and the results of our study was in consistence with those findings. However, Assmann et al. (31) reported similar resistance to dislodgement for both sealers. One of the differences between the two sealers is in the setting time, and MTA fillapex could not flow out, form the sealer tag and bond to the canal irregularities as a result of faster setting time. In addition, there is the possibility of formation of strong covalent bonds between exposed amino groups of dentin collagen and open epoxy rings in AH Plus sealer (27).

Disinfection of the root canal space is one of the main goals of root canal therapy and is necessary for healing of periapical diseases or for preventing them (32). PDT and antibacterial NPs are newly introduced root canal disinfectinion methods. Several studies have been conducted on the antimicrobial effectiveness of these two but their impact on the dentin surface and adhesion of sealers has been less attended in this studies

$\mathrm{Ag}$ nanoparticles prevent cell proliferation in the ionized form and cause cellular lysis (12), and $\mathrm{ZnO}$ nanoparticles destroy the bacteria by disrupting cell membranes (13). These particles together with $(\mathrm{Ag} / \mathrm{ZnO})$ have a higher antibacterial activity (33). PDT is a method in which by irradiating light with a specific wavelength to light-sensitive material (photosensitizer), localized in targeted tissue, become activated and produce free radicals which cause oxidative damage of target cells (34). The disadvantage in both methods is the reduced effectiveness in the presence of pulp tissue and root canal content (35), and therefore in this study they were used as an adjunct and final step in root canal disinfection process. Our study showed that NPs had a negative effect on AH plus adhesion to radicular dentin. The deposition of NPs on the radicular dentin surface may reduces the contact area and the adhesion of sealer to dentin. They also block the orifices of the dentinal tubules and prevent the sealer tag formation. Further studies are needed to investigate the effects of nanoparticles on the surface properties of dentin and their reaction with canal filling materials.

Conversely PDT showed positive effect on the adhesion of the both sealers in this study. Increased adhesion of resin-based sealers to dentin after treatment with laser Er:YAG (36) Nd: YAG (37) and the 980-nm diode laser (38) have been reported previously. The results of our study using diode laser for PDT also are consistent with these results. However Ok et al. (26) who had used the LED for PDT reported that it had no significant effect on bond strength of AH Plus sealer. The difference could be attributed to the light sources. Laser irradiation to the dentin surface removes the smear layer and also causes changes in the morphology of the dentin $(39,40)$, thus, by increasing the irregularities and dentinal contact surface, the adhesion of sealer to dentin increases. These changes have been observed with an electron microscopy $(39,40)$. SEM evaluation in the present study revealed deeper penetration of $\mathrm{AH}$ plus into the dentinal tubules with PDT using laser (Fig. 3F)

Tooth discoloration is an adverse effect of PDT when toluidine blue is used as photosensitizers. The alcohol compounds can be used to clean up this color material but given that a resin-based sealer was used in this study, no attempt was made to clean the dye because of the possibility of interference, which is of the limitations of this study. Studies should be continue to finding a way to benefit from the potential long term antibacterial effects of $\mathrm{Ag} / \mathrm{ZnO}$ NPs despite the effect on push-out test observed in this study.

\section{Conclusions}

Sealer adhesion to dentin is influenced by the type of sealer and canal disinfection method used. Considering the limitations of this study PDT is an appropriate adjunctive disinfection method for endodontic treatment because in addition to anti-bacterial property it can increase the adhesion of sealer to dentin.

\section{References}

1. Siqueira JF Jr, Rôças IN, Lopes HP, de Uzeda M. Coronal leakage of two root canal sealers containing calcium hydroxide after exposure to human saliva. Journal of Endodontics. 1999;25:14-6.

2. Hashem AAR, Ghoneim AG, Lutfy RA, Fouda MY. The effect of different irrigating solutions on bond strength of two root canal-filling systems. Journal of Endodontics. 2009;35:537-40.

3. Teixeira CS, Alfredo E, Thomé LHdC, Gariba-Silva R, Silva-Sousa YTC, Sousa-Neto MD. Adhesion of an endodontic sealer to dentin and gutta-percha: shear and push-out bond strength measurements and SEM analysis. Journal of Applied Oral Science. 2009;17:129-35.

4. Ayrancı LB, Köseoğlu M. The Evaluation of the Effects of Different Irrigating Solutions and Laser Systems on Adhesion of Resin-Based Root Canal Sealers. Photomedicine and laser surgery. 2014;32:152-9. 5. Gonçalves L, Silva-Sousa YTC, Raucci Neto W, Teixeira CS, SousaNeto MD, Alfredo E. Effect of different irrigation protocols on the radicular dentin interface and bond strength with a metacrylate-based endodontic sealer. Microscopy research and technique. 2014;77:44652 .

6. Nagas E, Uyanik MO, Eymirli A, Cehreli ZC, Vallittu PK, Lassila $\mathrm{LV}$, et al. Dentin moisture conditions affect the adhesion of root canal sealers. Journal of Endodontics. 2012;38:240-4.

7. Ozkocak I, Sonat B. Evaluation of effects on the adhesion of various root canal sealers after Er: YAG laser and irrigants are used on the dentin surface. Journal of endodontics. 2015;41:1331-6.

8. Reyhani MF, Ghasemi N, Rahimi S, Milani AS, Mokhtari H, Shakouie S, et al. Push-out bond strength of Dorifill, Epiphany and MTA-Fillapex sealers to root canal dentin with and without smear layer. Iran Endod J. 2014;9:246-50. 
9. Lotfi M, Vosoughhosseini S, Saghiri MA, Zand V, Ranjkesh B, Ghasemi N. Effect of MTAD as a final rinse on removal of smear layer in ten-minute preparation time. Journal of endodontics. 2012;38:1391-4. 10. Peters L, Van Winkelhoff AJ, Buijs J, Wesselink P. Effects of instrumentation, irrigation and dressing with calcium hydroxide on infection in pulpless teeth with periapical bone lesions. International Endodontic Journal. 2002;35:13-21.

11. Pagonis TC, Chen J, Fontana CR, Devalapally H, Ruggiero K, Song X, et al. Nanoparticle-based endodontic antimicrobial photodynamic therapy. Journal of endodontics. 2010;36:322-8.

12. Prabhu S, Poulose EK. Silver nanoparticles: mechanism of antimicrobial action, synthesis, medical applications, and toxicity effects. International Nano Letters. 2012;2:32.

13. Zhang L, Jiang Y, Ding Y, Povey M, York D. Investigation into the antibacterial behaviour of suspensions of $\mathrm{ZnO}$ nanoparticles $\mathrm{ZnO}$ nanofluids). Journal of Nanoparticle Research. 2007;9:479-89.

14. Chrepa V, Kotsakis GA, Pagonis TC, Hargreaves KM. The effect of photodynamic therapy in root canal disinfection: a systematic review. Journal of endodontics. 2014;40:891-8.

15. Souza LC, Brito PR, de Oliveira JCM, Alves FR, Moreira EJ, Sampaio-Filho HR, et al. Photodynamic therapy with two different photosensitizers as a supplement to instrumentation/irrigation procedures in promoting intracanal reduction of Enterococcus faecalis. Journal of Endodontics. 2010;36:292-6.

16. Akcay M, Arslan H, Topcuoglu HS, Tuncay O. Effect of calcium hydroxide and double and triple antibiotic pastes on the bond strength of epoxy resin-based sealer to root canal dentin. Journal of endodontics. 2014;40:1663-7.

17. Eldeniz AU, Erdemir A, Belli S. Shear bond strength of three resin based sealers to dentin with and without the smear layer. Journal of Endodontics. 2005;31:293-6.

18. Topçuoğlu HS, Tuncay Ö, Demirbuga S, Dinçer AN, Arslan H. The Effect of Different Final Irrigant Activation Techniques on the Bond Strength of an Epoxy Resin-based Endodontic Sealer: A Preliminary Study. Journal of endodontics. 2014;40:862-6.

19. Rawtiya M, Verma K, Singh S, Munuga S, Khan S. MTA-based root canal sealers. J Orofac Res. 2013;3:16-21.

20. Teixeira FB, Teixeira EC, Thompson JY, Trope M. Fracture resistance of roots endodontically treated with a new resin filling material. The Journal of the American Dental Association. 2004;135:646-52.

21. Huffman B, Mai S, Pinna L, Weller R, Primus C, Gutmann J, et al. Dislocation resistance of ProRoot Endo Sealer, a calcium silicate-based root canal sealer, from radicular dentine. International Endodontic Journal. 2009;42:34-46.

22. Rached-Junior FJA, Souza-Gabriel AE, Alfredo E, Miranda CES, Silva-Sousa YTC, Sousa-Neto MD. Bond strength of Epiphany sealer prepared with resinous solvent. Journal of endodontics. 2009;35:251-5.

23. Shokouhinejad N, Gorjestani H, Nasseh AA, Hoseini A, Mohammadi M, Shamshiri AR. Push-out bond strength of gutta-percha with a new bioceramic sealer in the presence or absence of smear layer. Australian Endodontic Journal. 2013;39:102-6.

24. Schwartz RS. Adhesive dentistry and endodontics. Part 2: bonding in the root canal system - the promise and the problems: a review. Journal of Endodontics. 2006;32:1125-34.

25. Goracci C, Tavares AU, Fabianelli A, Monticelli F, Raffaelli O, Cardoso PC, et al. The adhesion between fiber posts and root canal walls: comparison between microtensile and push-out bond strength measurements. European Journal of Oral Sciences. 2004;112:353-61. 26. Ok E, Ertas H, Saygili G, Gok T. Effect of photoactivated disinfection on bond strength of root canal filling. Journal of endodontics. 2013;39:1428-30.

27. Stelzer R, Schaller H-G, Gernhardt CR. Push-out bond strength of RealSeal SE and AH Plus after using different irrigation solutions. Journal of endodontics. 2014;40:1654-7.

28. Shokouhinejad N, Sabeti M, Gorjestani H, Saghiri MA, Lotfi M, Hoseini A. Penetration of Epiphany, Epiphany self-etch, and AH Plus into dentinal tubules: a scanning electron microscopy study. Journal of endodontics. 2011;37:1316-9.
29. Saleh IM, Ruyter IE, Haapasalo MP, Ørstavik D. Adhesion of endodontic sealers: scanning electron microscopy and energy dispersive spectroscopy. Journal of endodontics. 2003;29:595-601.

30. Sönmez I, Sönmez D, Almaz M. Evaluation of push-out bond strength of a new MTA-based sealer. European Archives of Paediatric Dentistry. 2013;14:161-6.

31. Assmann E, Scarparo RK, Böttcher DE, Grecca FS. Dentin bond strength of two mineral trioxide aggregate-based and one epoxy resinbased sealers. Journal of endodontics. 2012;38:219-21.

32. Nair P. On the causes of persistent apical periodontitis: a review. International endodontic journal. 2006;39:249-81.

33. Samiei M, Ghasemi N, Divband B, Balaei E, Hosien SBM, Divband A. Antibacterial efficacy of polymer containing nanoparticles in comparison with sodium hypochlorite in infected root canals. Minerva stomatologica. 2015;64:275-81.

34. Siddiqui SH, Awan KH, Javed F. Bactericidal efficacy of photodynamic therapy against Enterococcus faecalis in infected root canals: a systematic literature review. Photodiagnosis and photodynamic therapy. 2013;10:632-43.

35. Shrestha A, Kishen A. The effect of tissue inhibitors on the antibacterial activity of chitosan nanoparticles and photodynamic therapy. Journal of endodontics. 2012;38:1275-8.

36. Pécora JD, Cussioli AL, Guerisoli D, Marchesan MA, Sousa-Neto MD, Brugnera Junior A. Evaluation of Er: YAG laser and EDTAC on dentin adhesion of six endodontic sealers. Braz Dent J. 2001;12:2730 .

37. Sousa-Neto M, Silva Coelho F, Marchesan M, Alfredo E, SilvaSousa Y. Ex vivo study of the adhesion of an epoxy-based sealer to human dentine submitted to irradiation with Er: YAG and Nd: YAG lasers. International Endodontic Journal. 2005;38:866-70.

38. Alfredo E, Silva S, Ozório J, Sousa-Neto M, Brugnera-Júnior A, Silva-Sousa Y. Bond strength of AH Plus and Epiphany sealers on root dentine irradiated with $980 \mathrm{~nm}$ diode laser. International endodontic journal. 2008;41:733-40.

39. Sousa-Neto MD, Marchesan MA, Pécora JD, Junior AB, SilvaSousa YT, Saquy PC. Effect of Er: YAG laser on adhesion of root canal sealers. Journal of endodontics. 2002;28:185-7.

40. Türkmen C, Günday M, Karaçorlu M, Başaran B. Effect of CO 2, Nd: YAG, and ArF excimer lasers on dentin morphology and pulp chamber temperature: an in vitro study. Journal of endodontics. 2000;26:644-8.

\section{Conflicts of Interest}

The authors declare they have no conflicts of interest. 